03

\title{
Об одном механизме влияния неоднородностей поля силы тяжести на динамику атмосферы
}

\author{
(С) Л.Х. Ингель ${ }^{1,2}$, А.А. Макоско 2 \\ ${ }^{1}$ Научно-производственное объединение „Тайфунн“, \\ 249038 Обнинск, Россия \\ ${ }^{2}$ Институт фозики атмосферы им. А.М. Обухова РАН, \\ 119017 Москва, Россия \\ e-mail: lev.ingel@gmail.com
}

(Поступила в Редакцию 11 июля 2016 г. В окончательной редакции 7 февраля 2017 г.)

Показано, что при учете теплообмена горизонтальные неоднородности поля силы тяжести приводят к возникновению динамических возмущений (течений) в стратифицированной по плотности среде. Амплитуда горизонтальной составляющей возникающих течений пропорциональна произведению амплитуды отклонений геоида от общего земного эллипсоида и частоты плавучести.

DOI: 10.21883/JTF.2017.09.44903.1983

\section{Введение}

В современных моделях геофизической гидродинамики поле силы тяжести обычно принимается однородным и описывается с использованием единственного параметра $g$. Между тем известно, что на среднюю силу тяжести у поверхности Земли накладывается широкий спектр аномалий (неоднородностей) силы тяжести (АСТ). Это связано, прежде всего, с неоднородностями распределения массы в земной коре. Амплитуды вариаций силы тяжести, конечно, очень малы по сравнению со средним значением $g$. Но существенно, что при наличии таких неоднородностей появляется составляющая силы тяжести, тангенциальная по отношению к общему земному эллипсоиду. В плоских мезомасштабных моделях, в которых используются декартовы координаты (,f $f$-плоскость“, „бета-плоскость“ $[1,2])$, это означает необходимость учета дополнительных объемных неоднородных сил с горизонтальной составляющей. К таким составляющим динамика атмосферы и водоемов весьма чувствительна. В высокоаномальных регионах тангенциальные („горизонтальные“) составляющие силы тяжести на масштабах порядка $100 \mathrm{~km}$ могут превышать значения $100 \mathrm{mGal}\left(10^{-3} \mathrm{~m} / \mathrm{s}^{2}\right)$ [3,4]. Тем самым в уравнениях динамики атмосферы они могут быть сравнимыми по порядку величины, например, с горизонтальными силами градиента давления в циклонах умеренных широт и другими слагаемыми, необходимость учета которых не вызывает сомнений.

По меньшей мере с 70-х годов в геофизической литературе неоднократно высказывались предположения о возможности существенного влияния неоднородностей поля силы тяжести (НПСТ) на динамику некоторых атмосферных процессов (см., например, [4-7]). В частности, согласно [5], некоторые натурные данные указывают на возможное влияние АСТ на зарождение тропических циклонов (ТЦ). В последнее время в работах $[8-10]$ выполнен более репрезентативный статистический анализ, относящийся к возможному влиянию АСТ на ТЦ.
Имеется также некоторый опыт численного моделирования [11-13]; в этих работах сделаны выводы о возможности заметных атмосферных эффектов НПСТ. Но существует потребность в достаточно строгих и прозрачных аналитических моделях, без которых трудно добиться уверенного понимания физических механизмов влияния НПСТ на динамику атмосферы.

Известна теорема, согласно которой в покоящейся жидкой (газообразной) среде изобары и изопикны (поверхности равной плотности) должны совпадать с эквипотенциальными поверхностями [14]. Если изобары совпадают с поверхностями равного потенциала силы тяжести, то это означает, что сила градиента давления в каждой точке и в каждом направлении компенсирует силу тяжести. Поэтому существует статическое решение. В этой связи распространено мнение, что неоднородности поля силы тяжести лишь несколько „деформируют“, „искривляют“ состояние гидростатического равновесия, но не влияют на поле движения.

Но авторы обратили внимание на существование физических механизмов, которые, вообще говоря, должны нарушать это статическое равновесие. В частности, баланс сил тяжести и градиента давления может нарушаться при наличии фонового горизонтального течения [15]. В настоящей работе рассматривается другой механизм, связанный с учетом теплообмена в рассматриваемой среде (вывод [14] о полной взаимной компенсации сил тяжести и градиента давления относится к идеальной среде и не учитывает возможности теплообмена).

Геометрия задачи схематически пояснена на рис. 1. Через $x, z$ обозначены горизонтальная и вертикальная оси координат соответственно (для простоты ограничиваемся двумерной задачей). Штриховыми линиями обозначены эквипотенциальные поверхности, с которыми, согласно упомянутой теореме, совпадают изобары, изопикны и, следовательно, изотермы в идеальной среде. В области аномалии силы тяжести эти поверхности деформированы (в случае отрицательной АСТ изогнуты 


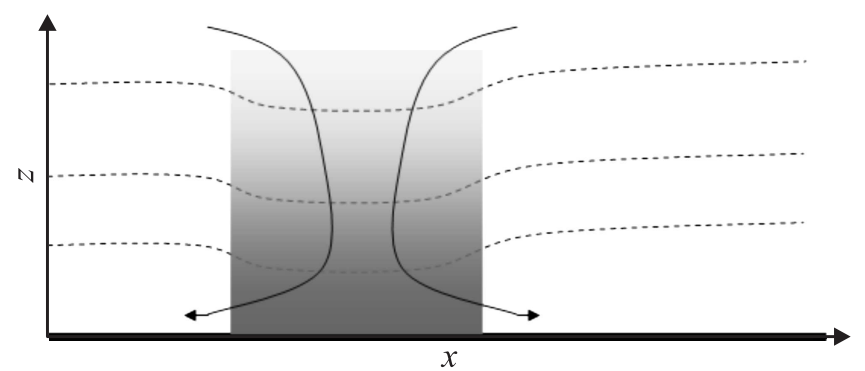

Pис. 1. Геометрия задачи (пояснено в тексте). Затушевана нижняя часть области аномалии силы тяжести, подверженная термическому влиянию нижней границы. Сплошные линии схематически изображают течения, возникающие вследствие связанного с теплообменом нарушения гидростатического баланса.

вниз). В этой области должна существовать некоторая температурная неоднородность (в атмосфере в качестве соответствующей переменной удобнее использовать так называемую потенциальную температуру $[1,2])$. При учете теплообмена с окружающей средой (в атмосфере существен прежде всего турбулентный обмен c нижней границей - подстилающей поверхностью) эта неоднородность должна в какой-то мере релаксировать (на рисунке затушевана область, подверженная влиянию теплообмена с нижней границей, температура которой предполагается фиксированной). Это, в свою очередь, должно отражаться на поле давления (при изменении температуры меняется вес столба среды). Таким образом, учет теплообмена приводит к изменениям пространственного распределения поля давления и тем самым к нарушению вышеупомянутого баланса силы тяжести и градиента давления. Тангенциальная (горизонтальная) составляющая силы тяжести теперь (с учетом теплообмена) компенсируется не в полной мере, а это означает возникновение течений. В этом принципиальное отличие от случая однородной силы тяжести, в котором учет вертикального теплообмена меняет лишь вертикальные распределения полей температуры, плотности и давления и может не приводить к возникновению нескомпенсированных горизонтальных сил.

Если рассматривать неоднородности поля силы тяжести с горизонтальными масштабами порядка $100-1000 \mathrm{~km}$, то эти масштабы много больше характерной толщины атмосферы. Поэтому наиболее существенна диффузия тепла в вертикальном направлении. Если в простейшей модели предполагать температуру горизонтальной нижней границы (подстилающей поверхности) фиксированной и постоянной, то эта граница при учете теплообмена, очевидно, будет влиять на поле температуры в нижнем слое среды. Изотермы и изобары вблизи границы при этом уже не совпадают с эквипотенциальными поверхностями; баланс горизонтальных сил нарушается в пользу вариаций силы тяжести. Ниже представлен простейший вариант линейной стационарной модели возникающих при этом течений.

\section{Постановка задачи}

Математическая задача в ряде отношений аналогична известным исследованиям течений, возникающих в стратифицированной среде над неоднородно нагретой горизонтальной поверхностью (см., например, $[16,17])$. Но там возмущения в среде связаны с неоднородными краевыми условиями на нижней границе, а в настоящем случае эти условия однородны, но неоднородна система уравнений гидротермодинамики - в ней присутствует дополнительная горизонтально-неоднородная сила, связанная с неоднородностью силы тяжести.

Для обобщения стандартных уравнений динамики с учетом неоднородностей поля силы тяжести введем в эти уравнения дополнительные силы (ускорения) $g_{x}(x, z), g_{z}(x, z)$ - горизонтальную и вертикальную составляющие АСТ (помимо обычно рассматриваемой постоянной силы тяжести, обозначаемой через $g$ ) [15]. Из свойств гравитационного потенциала следует соотношение

$$
\frac{\partial g_{x}}{\partial z}=\frac{\partial g_{z}}{\partial x}
$$

В качестве первого шага имеет смысл рассмотреть задачу без учета кориолисовых ускорений, роль которых мала, например, в низких широтах.

Соответствующая линеаризованная система уравнений гидротермодинамики для стационарной задачи в приближении Буссинеска имеет вид

$$
\begin{gathered}
0=-\frac{1}{\bar{\rho}} \frac{\partial p}{\partial x}+v \Delta_{2} u+g_{x}, \\
0=-\frac{1}{\bar{\rho}} \frac{\partial p}{\partial z}+v \Delta_{2} w-g \frac{\rho^{\prime}}{\bar{\rho}}+g_{z}, \quad \frac{\partial u}{\partial x}+\frac{\partial w}{\partial z}=0, \\
\gamma w=\kappa \Delta_{2} \theta, \quad \rho^{\prime}=-\bar{\rho} \alpha \theta .
\end{gathered}
$$

Здесь $u, w-$ составляющие возмущения поля скорости вдоль осей $x, z$ соответственно, $p^{\prime}, \rho^{\prime}, \theta-$ возмущения давления, плотности и потенциальной температуры, $v-$ кинематический коэффициент вязкости, $\kappa-$ коэффициент температуропроводности (предполагаются постоянными), $\gamma>0$ - фоновый вертикальный градиент потенциальной температуры (предполагается устойчивая фоновая стратификация плотности), $\alpha-$ коэффициент теплового расширения, $\bar{\rho}-$ средняя (отсчетная) плотность среды, $\Delta_{2}-$ символ двумерного лапласиана.

На нижней границе (подстилающей поверхности) предполагается выполнение условий непротекания и прилипания, а также фиксированной температуры (отсутствия температурных возмущений):

$$
u=w=0, \quad \theta=0 \quad \text { при } \quad z=0 .
$$

Вдали от поверхности предполагается выход на статический режим, существующий, согласно [14], при отсутствии теплообмена, т.е. без учета влияния подстилающей поверхности (горизонтальный теплообмен в рассматриваемой геометрии задачи незначителен). 
Последнее означает, что изобары, изопикны и изотермы вдали от нижней границы совпадают с эквипотенциальными поверхностями, а возмущения скорости затухают. Обозначим через $\Phi$ и $\eta$ соответственно отклонения потенциала силы тяжести и вертикальные отклонения эквипотенциальных поверхностей, связанные с неоднородностями поля силы тяжести. По определению $\eta=-\Phi / g=\int g_{x} d x / g$, где нижний предел интегрирования - „отсчетная“" точка, в которой упомянутые отклонения отсутствуют. Соответственно верхнее граничное условие для температурного возмущения имеет вид

$$
\theta \rightarrow-\gamma \eta=-\gamma \int g_{x} d x / g \quad \text { при } \quad z \rightarrow \infty .
$$

\section{Решение}

Исключая из системы уравнений все неизвестные, кроме $w$, получаем уравнение

$$
\Delta_{2}^{3} w+\frac{N^{2}}{\kappa v} \frac{\partial^{2} w}{\partial x^{2}}=0
$$

где $N=(\alpha g \gamma)^{1 / 2}$ - частота плавучести (частота Брента-Вяйсяля).

Удобно анализировать модель с синусоидальной зависимостью неоднородностей поля силы тяжести от горизонтальной координаты:

$$
g_{x}=G \exp (-k z) \cos k x, g_{z}=-G \exp (-k z) \sin k z,
$$

где $G$ - амплитуда, $k^{-1}$ - пространственный масштаб неоднородности соответственно. В этом случае решение также ищем в виде горизонтальной гармоники:

$$
u(x, z)=U(z) \cos k x, w(x, z)=W(z) \sin k x, \text { и т.д. }
$$

Уравнение (7) принимает вид

$$
\left(\frac{d^{2}}{d Z^{2}}-1\right)^{3} W=\mathrm{R} W, \quad \mathrm{R}=\frac{N^{2}}{\kappa v k^{4}} .
$$

Здесь введены безразмерная переменная $Z=k z$ и безразмерный параметр $\mathrm{R}<0$, с точностью до знака, являющийся некоторым аналогом числа Рэлея.

Решение последнего уравнения стандартным образом ищем в виде линейной комбинации экспонент типа $\exp (\sigma k z)$. Характеристическое уравнение имеет вид

$$
\left(\sigma^{2}-1\right)^{3}=\mathrm{R} .
$$

С учетом затухания при $z \rightarrow \infty$ решение для вертикальной скорости представляет собой линейную комбинацию трех экспонент

$$
w(x, z)=\sum_{j=1}^{3} C_{j} \exp \left(k \sigma_{j} z\right) \sin k x
$$

где отобраны корни $\sigma_{j}$ с отрицательными действительными частями (здесь предполагается, что эти корни различны). Учитывая уравнение неразрывности, имеем

$$
u(x, z)=\sum_{j=1}^{3} C_{j} \sigma_{j} \exp \left(k \sigma_{j} z\right) \cos k x .
$$

В выражениях для возмущений температуры и давления, как нетрудно проверить, присутствуют также слагаемые с четвертой экспонентой $\exp (-k z)$. Выражение для температурного возмущения можно представить в виде

$$
\theta(x, z)=\left[C_{4} \exp (-k z)+\frac{\gamma}{\kappa k^{2}} \sum_{j=1}^{3} \frac{C_{j}}{\sigma_{j}^{2}-1} \exp \left(k \sigma_{j} z\right)\right] \sin k x .
$$

При характерных для атмосферы значениях параметров на рассматриваемых достаточно больших горизонтальных масштабах безразмерное число R весьма велико. Например, при $N=10^{-2} \mathrm{~s}^{-1}, \kappa=v=1 \mathrm{~m}^{2} / \mathrm{s}$, $k=2 \cdot 10^{-5} \mathrm{~m}^{-1}$ (что соответствует длине горизонтальной полуволны около $150 \mathrm{~km}) \mathrm{R} \sim 10^{15}$. В этом случае корни характеристического уравнения $\sigma_{j}$ велики по абсолютной величине по сравнению с единицей, и в формулах (11), (14) можно пренебрегать единицей по сравнению с квадратами этих корней (физически это соответствует пренебрежимо малой роли горизонтального обмена). Три корня с отрицательными действительными частями:

$$
\sigma_{1} \approx-R^{1 / 6}, \quad \sigma_{2,3} \approx-R^{1 / 6} \exp \left( \pm i \frac{\pi}{3}\right) .
$$

В выражении (14) первая из экспонент убывает очень медленно по сравнению с остальными тремя. Поэтому из краевого условия (6) в сочетании с (8) следует

$$
C_{4} \approx-\frac{\gamma G}{k g} \text {. }
$$

Остальные коэффициенты $C_{j}$ находятся из системы трех уравнений, вытекающих из краевых условий (5):

$$
\sum_{j=1}^{3} C_{j}=0, \quad \sum_{j=1}^{3} \sigma_{j} C_{j}=0, \quad \sum_{j=1}^{3} C_{j} / \sigma_{j}^{2} \approx \frac{\kappa k G}{g} .
$$

Решение имеет вид

$C_{1} \approx \frac{1}{2} \frac{\kappa k G}{g} \mathrm{R}^{1 / 3}, \quad C_{2,3} \approx-\frac{1}{2 \sqrt{3}} \frac{\kappa k G}{g} \mathrm{R}^{1 / 3} \exp \left(\mp i \frac{\pi}{6}\right)$.

Отсюда вертикальная скорость возникающего течения

$$
\begin{aligned}
w \approx & \frac{1}{2} h k \Xi \sqrt{\frac{\kappa}{v}}\left[\exp \left(-\frac{z}{h}\right)-\frac{2}{\sqrt{3}} \exp \left(-\frac{1}{2} \frac{z}{h}\right)\right. \\
& \left.\times \cos \left(\frac{\sqrt{3}}{2} \frac{z}{h}+\frac{\pi}{6}\right)\right] \sin k x,
\end{aligned}
$$

где введены масштабы длины $h=\left(\kappa v / N^{2} k^{2}\right)^{1 / 6}=$ $=k^{-1} \mathrm{R}^{-1 / 6}$ (вертикальный масштаб течений, возникающих у подстилающей поверхности) и скорости

$$
\Xi=\frac{N}{k} \frac{G}{g}=N \eta
$$




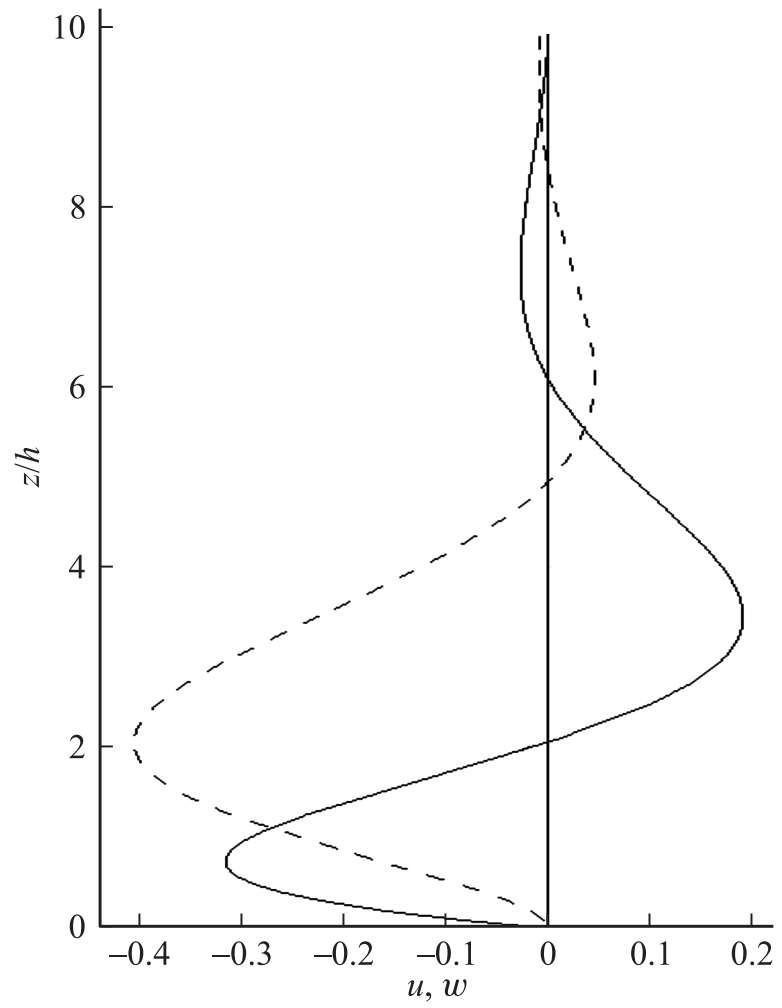

Рис. 2. Вертикальные профили $u$ (сплошная кривая, нормировано на $\Xi \sqrt{\kappa / v} / 2$ ) при $k x=\pi$ и $w$ (штриховая линия, нормировано на $\Xi k h \sqrt{\kappa / v} / 2)$, при $k x=3 \pi / 2$.

Для горизонтальной скорости получается выражение

$$
\begin{aligned}
u \approx & -\frac{1}{2} \Xi \sqrt{\frac{\kappa}{v}}\left[\exp \left(-\frac{z}{h}\right)-\frac{2}{\sqrt{3}} \exp \left(-\frac{z}{2 h}\right)\right. \\
& \left.\times \cos \left(\frac{\sqrt{3}}{2} \frac{z}{h}-\frac{\pi}{6}\right)\right] \cos k x .
\end{aligned}
$$

Вертикальные профили составляющих скорости представлены на рис. 2.

\section{Анализ решения}

Характерные амплитуды возмущений можно оценить из следующих простых соображений.

Когда изотермы совпадают с эквипотенциальными поверхностями, они в области аномалии смещены по вертикали на величину $\eta$. Следовательно, вдали от подстилающей поверхности отклонения температуры в АСТ порядка - $\eta$. Эти отклонения температуры (плотности, давления) обеспечивают гидростатический баланс: вариации силы тяжести в каждой точке и в любом направлении уравновешены ,дополнительными“ отклонениями давления. Но этот баланс нарушается вблизи подстилающей поверхности, температура которой предполагается фиксированной. Вследствие теплообмена, вблизи подстилающей поверхности имеется слой среды, температура которого в большой степени контролируется этой поверхностью. Там температура близка к фиксированной температуре нижней границы, т.е. наличие этой границы препятствует возникновению упомянутых отклонений температуры (отклонению изотерм от горизонтали), и, следовательно, поле температуры (плотности) вблизи границы отличается от того, которое обеспечивает упомянутый баланс сил. Это отличие $\Delta \theta$ поля температуры от „равновесного“ (соответствующего теореме [14]) по абсолютной величине, очевидно, порядка $|\gamma \eta|$, что согласуется с полученным решением для отклонений температуры. Соответственно гидростатическое давление отличается от того, которое обеспечивает баланс горизонтальных сил на величину $|\Delta p| \sim|g h \Delta \rho| \sim|g h \alpha \bar{\rho} \Delta \theta|$. Здесь $h$ - толщина слоя, в который проникают диффузионные возмущения от подстилающей поверхности. Этот вертикальный масштаб давно известен $[16,18]$, он совпадает с определенной выше величиной $h=\left(\kappa v / N^{2} k^{2}\right)^{1 / 6}=k^{-1} \mathrm{R}^{-1 / 6}$. Далее можно оценить возникающую у поверхности в нижнем слое толщиной порядка $h$ несбалансированную горизонтальную силу $(1 / \bar{\rho}) \partial \Delta p / \partial x$. Приравняв ее вязкой силе $v \partial^{2} u / \partial z^{2} \sim v u / h^{2}$, можем оценить характерную горизонтальную скорость возникающего течения. Эти простые соображения легко приводят к оценке амплитуды возмущений скорости, которая с точностью до безразмерного коэффициента согласуется с решением (18). Таким образом, смысл полученного решения вполне прозрачен. Течение возникает в приповерхностном слое толщиной порядка $h$ из-за того, что вследствие диссипации температурных неоднородностей баланс горизонтальных сил нарушается в пользу силы тяжести.

Возникающие течения, видимо, могут быть наиболее заметными в приповерхностном слое, где стратификация плотности бывает достаточно сильной. Пусть, например, в таком слое градиент потенциальной температуры $\gamma=0.1 \mathrm{~K} / \mathrm{m}$, так что $N \approx 0.05 \mathrm{~s}^{-1}$. Если отклонение геоида $\eta=20 \mathrm{~m}$, то масштаб скорости возникающего горизонтального течения $\Xi \approx 1 \mathrm{~m} / \mathrm{s}$ (правда, согласно, (18), этот масштаб умножается на безразмерную функцию, амплитуда которой порядка 1/6).

Как видно из рис. 2, возмущения скорости с высотой осциллируют, затухая на вертикальных масштабах порядка $h$. Если $\kappa=v=1 \mathrm{~m}^{2} / \mathrm{s}, k=0.5 \cdot 10^{-5} \mathrm{~m}^{-1}$ (что соответствует длине горизонтальной полуволны около $600 \mathrm{~km})$, то $h \approx 300 \mathrm{~m}$. В области отрицательной АСТ (вблизи вертикали $x=3 \pi / 2$ ) у поверхности преобладают горизонтальное растекание (положительная горизонтальная дивергенция) и нисходящее движение. Это понятно, поскольку в указанной области баланс горизонтальных сил нарушен в пользу силы тяжести, которая и определяет направление горизонтального движения.

Интересно отметить слабую зависимость результатов от значений коэффициентов обмена. Амплитуды составляющих скорости $(17),(18)$ зависят только от $(\kappa / v)^{1 / 2}$. В распространенных моделях турбулентного обмена эта величина равна или близка к единице. В выражение для 
вертикального масштаба $h$ упомянутые коэффициенты входят в степени 1/6. С усилением турбулентного обмена возрастает толщина слоя $h$, в котором сказывается влияние температуры подстилающей поверхности. Поэтому возрастает соответствующее отклонение веса столба воздуха - амплитуда возмущения горизонтальной силы градиента давления. Но при этом увеличивается и турбулентная вязкость. Поэтому амплитуда скорости возникающего горизонтального течения практически не меняется. Эта амплитуда не зависит в явном виде и от горизонтального масштаба неоднородностей только от амплитуды отклонения эквипотенциальных поверхностей.

\section{Заключение}

В горизонтально-однородной среде учет теплообмена влияет лишь на вертикальные распределения температуры, плотности и давления. Представленный выше анализ демонстрирует эффект, на который ранее не обращалось внимания: при учете теплообмена горизонтальные неоднородности поля силы тяжести могут приводить к возникновению динамических возмущений (течений) в стратифицированной по плотности среде. Горизонтальная скорость возникающих течений пропорциональна произведению отклонения геоида от общего земного эллипсоида и частоты плавучести.

Работа выполнена при поддержке программы № 15 фундаментальных исследований президиума РАН.

\section{Список литературы}

[1] Педлоски Джс. Геофизическая гидродинамика. М.: Мир, 1984. T. 1. 398 c. (Pedlosky J. Geophysical fluid dynamics. Springer-Verlag, 1987. 710 p.)

[2] Гилл A.E. Динамика атмосферы и океана. Т. 1. М.: Мир, 1986. 397 c. (Gill A.E. Atmosphere-Ocean Dynamics. N.Y. et al: Academic Press, 1982).

[3] Цубои T. Гравитационное поле Земли. М.: Мир, 1982. $286 \mathrm{c}$.

[4] Макоско А.А., Панин Б.Д. Динамика атмосферы в неоднородном поле силы тяжести. СПб.: РГГМУ, 2002. 246 с.

[5] Садоков В.П. // Метеорол. и гидрол. 1971. № 4. С. 14-26.

[6] Рудяев Ф.И. // ДАН СССР. 1990. Т. 310. № 6. C. $1345-1348$.

[7] Борисенков Е.П. // Вестник СПб. ун-та. Сер. 7. Геология. География. 2004. Вып. 1. С. 46-57.

[8] Ярошевич М.И. // Докл. РАН. 2011. Т. 437. № 4. C. $548-542$.

[9] Ярошевич М.И. // Изв. РАН. Физика атмосферы и океана. 2013. T. 49. № 3. С. 279-284.

[10] Yaroshevich M.I. // Trop. Cyclone Res. Rev. 2013. Vol. 2 . N 2. P. $124-130$.

[11] Макоско А.А., Рубинштейн К.Г., Лосев В.М., Боярский Э.A. Математическое моделирование атмосферы в неоднородном поле силы тяжести. М.: Наука, 2007. 58 с.

[12] Бычкова В.И., Макоско А.А., Рубинштейн К.Г., Егорова Е.Н. // Метеорол. и гидрол. 2011. № 7. С. 16-31.
[13] Макоско А.А., Рубинштейн К.Г. // Докл. РАН. 2014. Т. 459. № 2. C. 237-242.

[14] Кочин Н.Е. Изменение температуры и давления с высотой в свободной атмосфере. Собр. соч. Т. 1. М.-Л.: Изд. AH CCCP. 1949. C. 530-591.

[15] Ингель Л.Х., Макоско А.А. // Изв. РАН. Физика атмосферы и океана. 2015. Т. 51. № 4. С. 448-454.

[16] Stommel H., Veronis G. // Tellus. 1957. Vol. 9. N 3. C. 401-407.

[17] Ингель Л.Х., Беляева М.В. // Инженер.-физ. журн. 2011. T. 84. № 4. C. 759-763.

[18] Ингель Л.Х., Калашник М.В. // УФН. 2012. Т. 182. № 4. C. 379-406. 